\title{
Air pollution and daily admissions for chronic obstructive pulmonary disease in six European cities: results from the APHEA project
}

\section{To the Editor:}

ANDERSON et al. [1] present the results of time series analysis of data from the Air Pollution and Health, a European Approach (APHEA) project on air pollution and daily admissions for chronic obstructive pulmonary disease (COPD) from six European cities. The meritorious attainment of the APHEA study group was the agreement of all members on a methodological protocol enabling them to consider their results collectively and to gain a large sample size [2].

Units of exposure in this study pertain to geographical areas rather than to individuals. Consequently, the APHEA study design can be characterized by the term "ecological study". Ecological studies have well-known shortcomings for different reasons that have been discussed extensively by Morgenstern [3] as well as by Piantadosi et al. [4] Nondifferential misclassification is implicated as there are fewer possibilities to reduce this source of bias compar- ed to other study designs. This is especially true in the APHEA study with regard to the fact that concentrations of air pollutants such as ozone in different regions of a city (centre, residential quarters, periphery) can vary broadly, as the authors also discuss. As is generally postulated for epidemiological studies, nondifferential misclassification is supposed by the authors to result in an underestimation of a potential effect. In contrast to this, BRENNER et al. [5] have shown that in ecological studies nondifferential bias leads to overestimation of exposure-disease associations. Therefore, BRENNER et al. [5] recommend treating results from ecological studies with extreme caution. In this context, the APHEA authors' conclusion, from analysis of their data resulting in relative risks (RRs) of 1.02-1.04 (though statistically significant), suggesting a causal relationship between air pollutants and daily admissions for COPD, seems to lack a serious consideration of the possible weaknesses of their study design and their implications.

In general, RRs at a level of 1.02 to 1.04 in epidemiological studies and especially in ecological studies are not a convincing argument for causal relationship. Limits of risk assessment for epidemiological methods are estimated at $\mathrm{RR}=2.0[6,7]$. Below this level, the effects of bias and confounding may exceed the suspected association with the supposed causative agent. From the $\beta$-values of the multivariate model of collective analysis in the APHEA study it can be calculated that a level of $R R=2$ would be reached by an increase of ozone of $884 \mu \mathrm{g} \cdot \mathrm{m}^{-3}$ and/or of black smoke or, alternatively, at an increase of total suspended particulates and/or $\mathrm{NO}_{2}$ by $1,750 \mu \mathrm{g} \cdot \mathrm{m}^{-3}$. This calculation demonstrates that, in the model presented, a convincing elevated risk would only be detected at de-grees of outdoor pollution that are fortunately far from realistic scenarios in European cities at the moment [8-12].

Another source of doubt in the results of the study may be the $50 \mu \mathrm{g} \cdot \mathrm{m}^{-3}$ increase in all pollutants investigated, ignoring that such an increase for each pollutant may not occur consistently and may have different effects.

As the authors describe, the time lags supposed between air pollution and health effects $(0,1,2$ days) were selected at each centre on the basis of the greatest effect produced in time series analysis rather than a priori. In the discussion, the "possibility that different environments and healthcare systems might be associated with different lags" is mentioned without any firm reference to the lags introduced into the models. What could explain differences found between the time lags for hospital admissions for acute exacerbation of COPD in the six cities? One would expect at least an effort to interpret the discrepancy of lags ( 0 versus 2 days) between Amsterdam and Rotterdam in three models including that for ozone. Otherwise, entering different lags into each model raises severe doubts on the credibility of the results.

However, all these uncertainties did not prevent the authors from making statements such as "the associations with ozone are the strongest and most consistent" or "the evidence points to a causal relationship (between pollutants and daily admissions for COPD)". This treatment of results of the epidemiological studies by specialists may compromise the reputation of epidemiological methodology and encourage general sceptism against epidemiology. This is to be regretted, because well-planned epidemiological studies based on accurate data can contribute effectively to scientific progress. Unfortunately, less effort is often spent on procurement of a reliable database than the elaborate and sophisticated statistical methods applied on this material afterwards. To examine the adverse health effects of pollution in a susceptible population, a prospective cohort study would certainly have led to more conclusive results. The widespread belief, in epidemiological studies, that it is possible to acquire useful results quickly and inexpensively by minimizing the efforts to obtain an accurate database, should be abandoned. If the database is unreliable, all further work may be in vain. Exaggeration of weak results, as is frequently practised, is not to be excused.

\section{References}

1. Anderson HR, Spix C, Medina S, et al. Air pollution and daily admissions for chronic obstructive pulmonary disease in 6 European cities: results from the APHEA project. Eur Respir J 1997; 10: 1064-1071.

2. Katsouyanni K, Schwartz J, Spix C, et al. Short term 
effects of air pollution on health: a European approach using epidemiologic time series data: the APHEA protocol. J Epidemiol Community Health 1996; 50 (Suppl 1): S12-S18.

3. Morgenstern H. Uses of ecologic analysis in epidemiologic research. Am J Public Health 1982; 72: 1336-1344.

4. Piantadosi S, Byar DP, Green SB. The ecological fallacy. Am J Epidemiol 1988; 127: 893-903.

5. Brenner H, Savitz DA, Jöckel KH, Greenland S. Effects of nondifferential exposure misclassification in ecologic studies. Am J Epidemiol 1992; 135: 85-95.

6. Überla KK. Boundaries of perception and knowledge for risk assessment in epidemiology. Int J Epidemiol 1990; 19 (Suppl. 1): S81-S83.

7. Taubes G. Epidemiology faces its limits. Science 1995. 269: 164-169.

8. Ponce de Leon A, Anderson HR, Bland JM, Strachan DP, Bower J. Effects of air pollution on daily admissions respiratory disease in London between 1987-88 and 199192. J Epidemiol Community Health 1996; 50 (Suppl. 1): S63-S70.
9. Dab W, Medina P Quénel P, et al. Short term respiratory health effects of ambient air pollution: results from the APHEA project in Paris. $J$ Epidemiol Community Health 1996; 50 (Suppl. 1): S42-S46.

10. Schouten JP, Vonk JM, de Graaf A. Short term effects of air pollution on emergency hospital admissions for respiratory disease: results from the APHEA project in two major cities in the Netherlands, 1977-1989. J Epidemiol Community Health 1996; 50 (Suppl. 1): S22-S29.

11. Vigotti MA, Rossi G, Bisanti L, Zanobetti A, Schwartz J. Short term effects of urban air pollution on respiratory health in Milan, Italy 1980-1989. J Epidemiol Coтmunity Health 1996; 50 (Suppl. 1): S71-S75.

12. Sunyer J, Castellsagué J, Saéz M, Tobias A, Antó JM. Air pollution and mortality in Barcelona. J Epidemiol Community Health 1996; 50 (Suppl. 1): S76-80.

\section{B. Hasford, G. Fruhmann}

Institute of Occupational and Environmental Medicine, University of Munich, Ziemssenstr. 1, D-80336 Munich, Germany.

\section{REPLY}

\section{From the authors:}

The letter from Hasford and Fruhmann raises a number of important points, some of which we can address and others which must remain a matter of debate.

The potential problems of ecological design are many, but do not preclude the use of such designs when these are the only feasible method of investigating important public health problems. In our study there were two ecological dimensions. The first was within each time series, since daily aggregated data were used. With this approach it is generally accepted that misclassification of exposure (which is highly likely) will under most conditions lead to an underestimation of the effect. The second ecological dimension was the spatial analysis across the various cities; it is the latter approach that is most relevant to the points raised in the letter from Hasford and Fruhmann. These apply only in part to ecological time series analysis and we do not believe that the misclassification constellations examined by BRENNER et al. [1], which produce a bias away from the null apply in our case. It is important to note that we observed homogeneous associations across different cities with different monitoring systems, healthcare systems and using different statistical teams.

The question of causality in observational studies will always be controversial, even when relative risks are high (as for example in smoking related disease), and it is obvious that the lower the relative risk, the higher the chance of a noncausal explanation for any association. However, we reject dogmatic approaches to consideration of causality (such as a relative risk of more than 2.0) or literal application of criteria which must be satisfied. Causal inference in the presence of small associations is clearly in need of further development but until better methods are available we must rely on the present judgmental approach. We freely acknowledged in our paper the possibility of noncausal explanations, but stand by our relatively cautious statement that the consistency of the associations "point" towards a causal relationship. As WeEd [2] has recently pointed out, epidemiologists have a moral obligation to consider public health recommendations when examining evidence for causality. We were aware that regulators would be considering our results in formulating air pollution guidelines and standards and in estimating public health impacts. It would have been unethical not to indicate to policy makers the possibility that these associations might represent an indication of low-level toxicity in the air.

The APHEA study was undertaken at a time when there was virtually no information about air pollution and daily health effects in Europe. This is one reason why an a priori decision was made to select the lag with the strongest effect. In fact, associations at other lags tended to be consistent in direction with the one selected. We do not agree with Hasford and Fruhmann that this policy "raises severe doubt on the credibility of the results", but shall address this issue formally in the next phase of the APHEA programme, which has recently been funded by the European Community. Hasford and Fruhmann are also concerned about the use of a $50 \mu \mathrm{g} \cdot \mathrm{m}^{-3}$ increment in pollutant for presenting the results. This was to facilitate cross city analysis, not to compare the toxicity of each pollutant. Our data enable the reader to calculate the effects of any increment that they may choose.

The contribution of small-effect epidemiology to public health policy will always be accompanied by debate between those who adopt the precautionary principle and those who reject any evidence if a possibility of noncausality exists. We have used available data and methodology, however imperfect, to help inform a current public health issue. Constructive criticism will help us do this better.

\section{References}

1. Brenner H, Savitz DA, Jöckel KH, Greenland S. Effects of nondifferential exposure misclassification in ecologic studies. Am J Epidemiol 1992; 135: 85-95.

2. Weed JL. On the use of causal criteria. Int J Epidemiol 1997; 26: $1137-1141$.

H.R. Anderson*, K. Katsouyanni**,C. Spix ${ }^{+}$

*Dept of Public Health Sciences, St George's Hospital Medical School, London, UK. **University of Athens Medical School, Department of Hygiene and Epidemiology, Athens, Greece. +GSF Forschungszentrum fur Umwelt und Gesundheit, Institute fur Epidemiologie, Neuherberg, Germany. 